\title{
A maximum likelihood estimate of the parameters of the FRB population
}

\author{
Siddhartha Bhattacharyya ${ }^{1 \star}$, Himanshu Tiwari ${ }^{2}$, Somnath Bharadwaj ${ }^{1}$ and Suman Majumdar ${ }^{2,3}$ \\ ${ }^{1}$ Department of Physics, Indian Institute of Technology, Kharagpur, India \\ ${ }^{2}$ Department of Astronomy, Astrophysics and Space Engineering, Indian Institute of Technology, Indore, India \\ ${ }^{3}$ Department of Physics, Blackett Laboratory, Imperial College, London SW7 2AZ, U.K.
}

15 September 2021

\begin{abstract}
We consider a sample of 82 non-repeating FRBs detected at Parkes, ASKAP, CHIME and UTMOST each of which operates over a different frequency range and has a different detection criteria. Using simulations, we perform a maximum likelihood analysis to determine the FRB population model which best fits this data. Our analysis shows that models where the pulse scatter broadening increases moderately with redshift $(z)$ are preferred over those where this increases very sharply or where scattering is absent. Further, models where the comoving event rate density is constant over $z$ are preferred over those where it follows the cosmological star formation rate. Two models for the host dispersion measure $\left(D M_{\text {host }}\right)$ distribution (a fixed and a random $D M_{\text {host }}$ ) are found to predict comparable results. We obtain the best fit parameter values $\alpha=-1.53_{-0.19}^{+0.29}, \bar{E}_{33}=1.55_{-0.22}^{+0.26}$ and $\gamma=0.77 \pm 0.24$. Here $\alpha$ is the spectral index, $\gamma$ is the exponent of the Schechter luminosity function and $\bar{E}_{33}$ is the mean FRB energy in units of $10^{33} \mathrm{~J}$ across $2128-2848 \mathrm{MHz}$ in the FRB rest frame.
\end{abstract}

Key words: transients: fast radio bursts, scattering.

\section{INTRODUCTION}

Fast radio bursts (FRBs) are milli-second duration, highly energetic $\left(\sim 10^{32}-10^{34} \mathrm{~J}\right.$ ) radio transients (Lorimer et al. 2007; Keane et al. 2011; Thornton et al. 2013). Several radio telescopes including Parkes ( eg. Price et al. 2018), ASKAP (eg. Bhandari et al. 2020), CHIME (eg. Amiri et al. 2021) and UTMOST (eg. Gupta et al. 2020) have each detected a considerable number of FRBs. Several of the detected FRBs are found to repeat (eg. Spitler et al. 2014; Amiri et al. 2021), however the non-repeating FRBs possibly form a separate population (Palaniswamy et al. 2018; Caleb et al. 2018; Lu $\&$ Piro 2019) . Here we only consider the non-repeating FRBs. The large dispersion measures (DMs), greater than the expected Milky Way contribution, strongly suggests that FRBs are extragalactic events. Direct redshift estimates are available only for a few of the observed FRBs which have been localised on the sky (eg. Macquart et al. 2020; Heintz et al. 2020). For most FRBs the redshifts are inferred from the observed DMs.

Several models have been proposed for the physical origin of the FRB emission, unfortunately there is no clear picture as yet. For example, the recently detected FRB 200428, which coincided with an X-ray burst from the Galactic magnetar SGR J1935 + 2154 (Ridnaia et al. 2021; Li et al. 2020; Tavani et al. 2021), suggests active magnetars as a source for some of the FRBs (Bochenek et al.

^ siddhartha@phy.iitkgp.ac.in
2020; Margalit et al. 2020). Platts et al. (2019) provides a summary of the different FRB models.

The spectral index $\alpha$ of the FRBs is not very well constrained at present. Macquart et al. (2019) have determined a mean value of $\alpha=-1.5_{-0.3}^{+0.2}$ for the sample of 23 FRBs detected at ASKAP. Houben et al. (2019) have proposed a lower limit $\alpha>-1.2 \pm-0.4$ considering the dearth of simultaneous detection of FRB 121102 at $1.4 \mathrm{GHz}$ and $150 \mathrm{MHz}$ respectively. The energy and redshift distribution of the FRBs is also not well understood. James et al. (2021) have modelled the FRB energy distribution using a simple power law . Zhang et al. (2021) have used the FRBs detected at Parkes and ASKAP to constrain the exponent for the energy distribution to a value -1.8 .

In an earlier work Bera et al. (2016) (hereafter Paper I) have modelled the FRB population and used this to make predictions for FRB detection at different telescopes. In a recent work Bhattacharyya \& Bharadwaj (2021) (hereafter Paper II) have used the two-dimensional Kolmogorov-Smirnov (KS) test to compare the FRBs observed at Parkes, ASKAP, CHIME and UTMOST with simulated predictions for different FRB population models. It is shown there that the parameter range $\alpha>4$ and $\bar{E}_{33}>60$ is ruled out with $95 \%$ confidence, here $\bar{E}_{33}$ is the mean energy of the FRBs population in units of $10^{33} \mathrm{~J}$. Paper II also predicts that "CHIME is unlikely to detect an FRB with extra-galactic dispersion measure $D M_{E x}$ exceeding $3700 \mathrm{pc} \mathrm{cm}^{-3}$ ", a prediction which is borne out in the recently released CHIME catalogue of 492 FRBs where the maximum value is $D M_{E x}=3006.7 \mathrm{pc} \mathrm{cm}^{-3}$. The modelling of the 
Table 1. Considering the four telescopes Parkes; (Burke-Spolaor \& Bannister 2014; Zhang et al. 2020, 2019; Keane et al. 2011; Lorimer et al. 2007; Champion et al. 2016; Petroff et al. 2019; Thornton et al. 2013; Ravi et al. 2015; Petroff et al. 2015, 2017; Keane et al. 2016; Bhandari et al. 2018a; Ravi et al. 2016; Price et al. 2018; Bhandari et al. 2018b) ASKAP; (Bannister et al. 2017; Shannon et al. 2018; Macquart et al. 2019; Agarwal et al. 2019; Qiu et al. 2019; Bhandari et al. 2019; Bannister et al. 2019; Prochaska et al. 2019; Shannon et al. 2019) CHIME; (Amiri et al. 2019) and UTMOST; (Caleb et al. 2017; Farah et al. 2018, 2019; Gupta et al. 2020), this shows the respective frequency range, limiting fluence $F_{l}$ and the number of FRBs detected there. Note that we have not included the 492 FRBs recently reported from CHIME. The value of $F_{l}$ depends on the threshold signal to noise ratio $(S / N)_{\text {th }}$ which is different for the different FRB surveys.To keep the analysis simple, we consider a fixed $(S / N)_{\text {th }}=10$. Further, ASKAP's sensitivity also differs based on the particular survey in which the FRB was detected. For simplicity, we have used a fixed value of $F_{l}$ for ASKAP.

\begin{tabular}{cccc}
\hline $\begin{array}{c}\text { Telescope } \\
\text { Name }\end{array}$ & $\begin{array}{c}\text { Frequency range } \\
(\mathrm{MHz})\end{array}$ & $\begin{array}{c}F_{l} \\
(\mathrm{Jy} \mathrm{ms})\end{array}$ & $\begin{array}{c}\text { Number of } \\
\text { non-repeating FRBs }\end{array}$ \\
\hline Parkes & $1157-1546$ & 0.5 & 29 \\
ASKAP & $1129-1465$ & 4.1 & 31 \\
CHIME & $400-800$ & 0.64 & 11 \\
UTMOST & $827-859$ & 3.25 & 11 \\
\hline
\end{tabular}

FRB population and simulations of Paper II have also been used in the present work, and these are summarized in the next section. In the present paper we have used a maximum likelihood analysis to estimate the parameters of the FRB population for which the predictions best match the observed FRB distribution.

\section{METHODOLOGY}

For our analysis we use 82 non-repeating FRBs detected by Parkes, ASKAP, CHIME and UTMOST which have each detected more than 10 non-repeating FRBs. The frequency range, limiting fluence and number of FRBs for these four telescopes are summarized in the Table 1. The recent CHIME data for 492 FRBs (Rafiei-Ravandi et al. 2021; Amiri et al. 2021) was released while this paper was being written, and we have not considered these here. Non-repeating FRBs have also been detected at several other telescopes, however the number of events at each of these telescopes is less than 10 which is not adequate for the statistical analysis performed here.

Each observed FRB is characterized by its dispersion measure $(D M)$, fluence $(F)$ and the pulse width $(w)$. Here we use the extragalactic component $D M_{\mathrm{Ex}}=D M-D M_{\mathrm{MW}}$ where the Milky Way contribution is calculated for each FRB using the NE2001 model (Cordes \& Lazio 2003). For the present work we have analysed the observed distribution of $D M_{\mathrm{Ex}}$ and $F$ values. For each telescope, we have gridded the the observed $D M_{\mathrm{Ex}}$ and $F$ range and calculated the number of observed FRBs $N_{a}$ at each grid point (Figure 1), the grid points here are labelled using $a$. Given the limited number of FRBs, it is necessary to use a very coarse grid for the present analysis.

We now briefly discuss our model for the FRB population. The model for the FRB population is presented in Paper I, and the reader is referred there for details. The intrinsic properties of each FRB event are characterised by three quantities $\alpha, E_{33}$ and $w_{i}$. Here the energy of the FRB pulse at any frequency $v$ is assumed to be proportional to $v^{\alpha}$ where $\alpha$ is the spectral index. For the present analysis we have assumed that all the FRBs have the same value of the spectral index $\alpha$. Here $E_{33}$ is the energy of the FRB (in units of

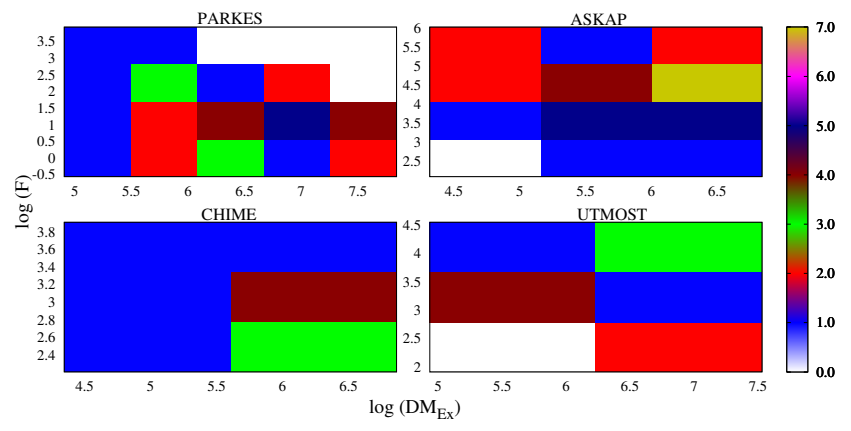

Figure 1. Considering the FRB observations at Parkes, ASKAP, CHIME and UTMOST, this shows the number of observed FRBs $N_{a}$ at each grid point. The color scale shows the number of FRBs at each grid.

$10^{33} \mathrm{~J}$ ) emitted in the frequency interval $2128 \mathrm{MHz}$ to $2848 \mathrm{MHz}$ at the rest frame of the source. $w_{i}$ here is the intrinsic pulse width of the FRB. Our earlier work (Paper II) shows that the results do not change much if we vary $w_{i}$ in the range $0.1 \mathrm{~ms}$ to $2.0 \mathrm{~ms}$, and here we have used a fixed value $w_{i}=1 \mathrm{~ms}$ for the entire analysis. In addition, each FRB also has a redshift $z$ and an angular position $\theta$ on the sky.

The FRB energy distribution is currently unknown. It is reasonable to assume that FRBs have a characteristic energy $\bar{E}_{33}$, and the distribution falls of rapidly beyond $\bar{E}_{33}$. For the analysis presented here we have assumed a Schechter luminosity function (Schechter 1976) for the energy distribution expressed as:

$n\left(E_{33}, z\right)= \begin{cases}\frac{n_{0}(z)}{\overline{E_{33}}} \exp \left(-\frac{E_{33}}{\bar{E}_{33}}\right) & \text { for } \gamma=0 \\ \frac{n_{0}(z)}{\bar{E}_{33}}\left(\frac{\gamma}{\Gamma(1+\gamma)}\right)\left(\frac{\gamma E_{33}}{\bar{E}_{33}}\right)^{\gamma} \exp \left(-\frac{\gamma E_{33}}{\bar{E}_{33}}\right) & \text { for } \gamma>0\end{cases}$

where $\bar{E}_{33}$ is the mean energy of the population, $\gamma$ is the exponent and $\Gamma(n)$ is the Gamma function. Here $n\left(E_{33}, z\right)$ is the FRB event rate per unit comoving volume per energy interval $d E_{33}$ and $n_{0}(z)$ is the FRB event rate per unit comoving volume. In our analysis, we have considered two possibilities for the event rate evolution with redshift, namely (a.) CER - constant event rate where $n_{0}(z)$ is independent of $z$ over the redshift range of our interest and (b.) SFR - where $n_{0}(z) \propto\left(0.015(1+z)^{2.7}\right) /\left(1+((1+z) / 2.9)^{5.6}\right)$ traces the star formation rate over cosmic time (Madau \& Dickinson 2014).

Considering an FRB located at redshift $z$, the observed pulse width $w=\sqrt{w_{\mathrm{cos}}^{2}+w_{\mathrm{DM}}^{2}+w_{\mathrm{sc}}^{2}}$ mainly has three contributions, namely (a) the cosmic expansion $w_{\cos }=w_{i}(1+z)$ - the intrinsic pulse width $w_{i}$, expanded by the cosmological expansion, (b) the dispersion broadening $w_{\mathrm{DM}}=\left(8.3 \times 10^{6} \mathrm{DM} \Delta v_{c}\right) / v_{0}^{3}$ - the residual dispersion broadening after the incoherent dedispersion of the FRB signal where $v_{0}$ is the observational frequency and $\Delta v_{c}$ is the channel width of the telescope, and (c) the scatter broadening $w_{\mathrm{sc}}$ - this is not well understood to date. Here $w$ is expressed in ms, and both $v_{0}$ and $\Delta v_{c}$ are expressed in MHz. In this work we have considered three scattering models, namely (a) Sc-I - based on the empirical fit of a large number of Galactic pulsar data provided by Bhat et al. (2004) and we have extrapolated this for the IGM, (b) Sc-II - this is a pure analytical model proposed by Macquart \& Koay (2013) considering the turbulent IGM, and (c) No-Sc - where there is no scattering and thus $w_{\mathrm{sc}}=0$. Our earlier work (Figure 1 of Paper-I) shows that for both Sc-I and -II, scattering dominates the total pulse width at redshifts $z>0.5$. Further, the pulse width increases very 
sharply with increasing redshift for Sc-I relative to Sc-II. The pulse width increases very slowly with redshift for No-Sc.

The contribution $D M_{\text {Host }}$ from the host galaxy is an unknown factor that enters FRB observations for most of the unlocalized FRBs. The value of $D M_{\text {Host }}$ is expected to vary from FRB to FRB depending on the host galaxy and the location of the FRB within it. However, the FRB detections suggest that $D M_{\text {Host }}$ may not exceed the value $100 \mathrm{pc} \mathrm{cm}^{-3}$ (Macquart et al. 2020). In addition to this, the observed DM will have another contribution $\left(D M_{\text {Halo }} \sim 50-80 \mathrm{pc} \mathrm{cm}^{-3}\right)$ from the Galactic halo (Prochaska \& Zheng 2019). Here we have absorbed the $D M_{\text {Halo }}$ contribution in $D M_{\text {Host }}$. In this work we consider two scenarios for $D M_{\text {Host }}$ namely (a) DM120 - where all FRBs have fixed $D M_{\text {Host }}=120 \mathrm{pc} \mathrm{cm}^{-3}$, and (b.) DMRand - where $D M_{\text {Host }}$ values are randomly drawn from a Gaussian distribution with mean $\overline{D M}_{\text {Host }}=120 \mathrm{pc} \mathrm{cm}^{-3}$ and root mean square value $\Delta D M_{\text {Host }}=15 \mathrm{pc} \mathrm{cm}^{-3}$. The $D M_{\text {Host }}$ distribution is truncated at 0 .

In summary, our model for the FRB population has three parameters $\alpha, \bar{E}$ and $\gamma$. Further, we have two models for the event rate distribution namely SFR and CER, three models for the scattering namely Sc-I, Sc-II and No-Sc, and two models for $D M_{\text {Host }}$ namely DM120 and DMRand.

We now briefly discuss how we use simulations to calculate $\mu_{a}$ (Figure 2) which is the model prediction for the mean number of FRBs expected to be detected at each grid point for any given telescope. These simulations are discussed in Paper II, and the interested readers are requested to refer to it for more details. We consider a comoving volume which extends up to $z_{\max }=5$, which is considerably higher than the highest redshift inferred for any of the FRB events included in our analysis. The angular extent of this comoving volume is equal to the full width half maxima (FWHM) of the primary beam of the telescope and this varies from telescope to telescope. We populate this comoving volume with $10^{6}$ randomly located FRBs whose mean comoving number density follow $n_{0}(z)$. This provides the comoving distance $r$ and angular position $\theta$ for each simulated FRB. We consider the $\Lambda C D M$ cosmology (Aghanim et al. 2020) to calculate $z$ from $r$. The energy $E_{33}$ of each FRB is randomly drawn from the distribution in eq. (1). We calculate $D M_{\mathrm{Ex}}, F$ and $w$ for each simulated FRB. The telescope can only detect an FRB if $F \times \sqrt{1 \mathrm{~ms} / w} \geq F_{l}$ where $F_{l}$ is the limiting fluence of the telescope which depends on the threshold signal to noise ratio $(S / N)_{\text {th }}$. Considering $(S / N)_{\text {th }}=10$, Table 1 lists the value of $F_{l}$ for the four telescopes considered here. We determine the fraction of observable events corresponding to each $\left(D M_{\mathrm{Ex}}, F\right)$ grid point and multiply this with the total number of FRBs actually observed by the telescope to calculate $\mu_{a}$ which is the mean number of FRB's expected in each grid point for the particular model under consideration. Figure 2 shows $\mu_{a}$ predicted for a particular model $\alpha=-1.53, \bar{E}_{33}=1.55$ and $\gamma=0.77$ with ScII, CER and DM120. We have compared the simulated predictions $\left(\mu_{a}\right)$ with the actual observation $\left(N_{a}\right)$ to identify preferred models for the FRB population.

We have used a Bayesian inference framework to constrain the parameters $\alpha, \bar{E}_{33}$ and $\gamma$ for all FRB models discussed here. The likelihood is estimated using the Poisson statistic. Considering the $a^{\text {th }}$ grid the probability of getting $N_{a}$ for a given $\mu_{a}$ is expressed as

$P_{a}=\frac{\mu_{a}^{N_{a}} \exp \left(-\mu_{a}\right)}{N_{a} !}$

where we assume that the likelihood $l_{a}$ is proportional to the probability, i.e. $l_{a} \propto P_{a}$. In this analysis we choose the proportionality

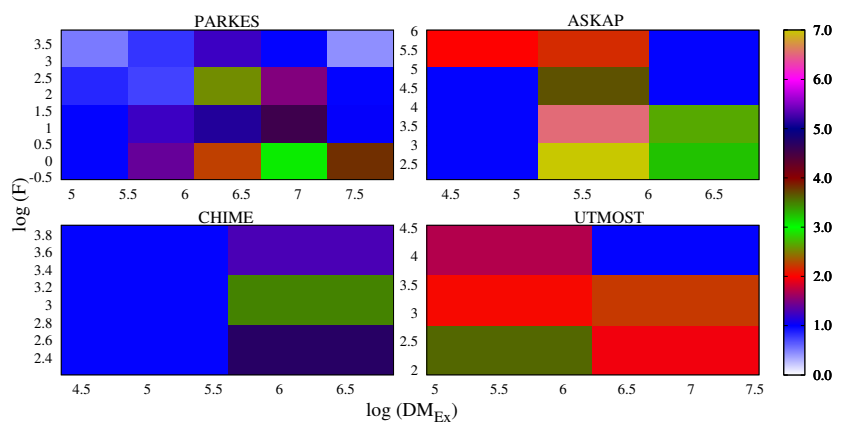

Figure 2. Considering Parkes, ASKAP, CHIME and UTMOST, this shows $\mu_{a}$ the mean number of FRBs predicted at each grid point for a model with $\alpha=-1.53, \bar{E}_{33}=1.55 \mathrm{~J}$ and $\gamma=0.77$ with Sc-II, CER and DM120. The color scale shows the number of FRBs at each grid.

constant to be 1 . Considering all the grid points, the logarithmic value of the total likelihood is given by

$\log (\mathcal{L})=\sum_{a} \log \left(l_{a}\right)=\sum_{a}\left[N_{a} \log \left(\mu_{a}\right)-\mu_{a}-\log \left(N_{a} !\right)\right]$

where the summation is taken over all the grid points. Our aim here is to probe efficiently the maximum likely region of the parameter space using a Markov-Chain-Monte-Carlo (MCMC) algorithm. It has been demonstrated by Autcha (2014) that in case of a Poissonian likelihood this can done efficiently by estimating the loss function:

$L\left(\mu_{a}, N_{a}\right)=-\sum_{a}\left[\mu_{a}-N_{a}\right]^{T} \cdot\left[\mu_{a}-N_{a}\right]$

at each step of the random walker in the parameter space. The evaluation of this loss function at each step of the random walk can efficiently guide the random walker towards the most likely region of the parameter space. For a detailed discussion on this, the interested readers are referred to Autcha (2014). In this work we have used the Python-based package Emcee ${ }^{1}$ (Foreman-Mackey et al. 2013)- an Affine invariant MCMC ensemble sampler (Goodman \& Weare 2010) to perform the exploration of the parameter space. For a specific FRB model and the given set of model parameters $\left(\alpha, \bar{E}_{33}, \gamma\right)$ we first predict $\mu_{a}$ and then using $N_{a}$ and $\mu_{a}$ we estimate the loss function $L\left(\mu_{a}, N_{a}\right)$ that eventually leads to estimation of the $\log (\mathcal{L})$ of the region around the maximum likelihood. For this analysis, we have used a standard MCMC chain of 20,000 samples with 40 random walkers. We discarded the initial 10 per cent of the 20,000 samples as the burnin steps. We have varied our parameter search between the range $\alpha \rightarrow(-30.0,+30.0), \bar{E}_{33} \rightarrow(0.01,20.00), \gamma \rightarrow(0.0,6.0)$, and assumed a uniform prior while doing the estimations.

\section{RESULTS}

Table 2 shows the best fit parameter values for all the models which we have considered here, the maximum values of log-likelihood $\log (\mathcal{L})$ are also shown alongside for reference. Considering the models with Sc-I, comparing the $\log (\mathcal{L})$ values we see that the models with CER are preferred over the SFR models. The same feature is also seen for the other scattering models. Guided by this, we exclude the SFR models and focus entirely on the CER models

\footnotetext{
1 Publicly available at: https://pypi.org/project/emcee/
} 
for the subsequent discussion. Comparing the different scattering models next, we find that Sc-II is preferred over both Sc-I and NoSc. Restricting our attention to Sc-II with CER, we find that DM120 and DMRand have comparable $\log (\mathcal{L})$ values. Although the $\log (\mathcal{L})$ value is slightly larger for DM120, the difference between DM120 and DMRand is very small in comparison to the difference with all the other models.

Considering Sc-II with CER, Figure 3 shows a corner plot of the parameters $\alpha, \bar{E}_{33}$ and $\gamma$ for both DM120 and DMRand. We first consider the three plots which show the joint distribution of pairs of these parameters. A visual inspection shows that in all cases the $68 \%$ and $95 \%$ confidence intervals for DM120 largely overlap with those for DMRand. Further, the orientation of the confidence intervals indicates that the constraints on the three parameters $\alpha$, $\bar{E}_{33}$ and $\gamma$ are largely uncorrelated. For DMRand for $(\alpha, \gamma)$ we notice that the $68 \%$ confidence interval is divided into two disconnected regions both of which are enclosed within the $95 \%$ confidence interval. Here also one of the region dominates whereas the other has a very low probability associated with it. Considering the two dimensional parameter plots, we see that in all cases our analysis imposes tight constraints on the joint distribution of the parameters $\alpha, \bar{E}_{33}$ and $\gamma$. We now consider the marginalised one dimensional plots which correspond to the best fit parameter values and the $68 \%$ confidence intervals reported in Table 2. We find that $\alpha$ is well constrained by our analysis with $\alpha=-1.53_{-0.19}^{+0.29}$ and $-1.57_{-0.24}^{+0.26}$ for DM120 and DMRand respectively. Similarly, we find that $\bar{E}_{33}$ is well constrained with $\bar{E}_{33}=1.55_{-0.22}^{+0.26}$ and $1.46_{-0.18}^{+0.25}$ for DM120 and DMRand respectively. We also find that $\gamma$ is well constrained with $\gamma=0.77 \pm 0.24$ and $0.988_{-0.102}^{+0.058}$ for DM120 and DMRand respectively. For $\alpha, \bar{E}_{33}$ and $\gamma$, the probability distributions are dominated by a single peak. Further, we see that the probability distributions for DM120 and DMRand have considerable overlap which indicates that the $\alpha, \bar{E}_{33}$ and $\gamma$ values estimated for these two models are consistent with one another.

\section{CONCLUSIONS}

In this analysis we have considered two distinct scenarios, one where the comoving FRB event rate evolves with redshift following the star formation rate and another where it is constant independent of redshift. Our analysis shows that the models with a constant event rate are preferred over the models where the event rate follows the star formation rate. The pulse broadening due to scattering in the IGM is not clearly understood at present. Here we have considered three possibilities. In the first model, based on Bhat et al. (2004) which provides an empirical fit of a large number of Galactic pulsar data, the scattering pulse width increases very sharply with increasing redshift. The second one is a purely theoretical model proposed by Macquart \& Koay (2013) considering the turbulent IGM. In this model the scattering pulse has a modest increase with increasing redshift. The third model assumes no scattering. Our analysis shows that the second model where there is a modest increase in pulse width with increasing redshift is preferred to the model where this increase is much steeper or the model where there is no scattering. The contribution to the dispersion measure from the host galaxies $D M_{\text {Host }}$ is another quantity which affects FRB detections. Here we consider two models, one where all the FRBs have fixed $D M_{\text {Host }}=120 \mathrm{pc} \mathrm{cm}^{-3}$, and another where the $D M_{\text {Host }}$ values are randomly drawn from a truncated Gaussian distribution with mean $\overline{D M}_{\text {Host }}=120 \mathrm{pc} \mathrm{cm}^{-3}$ and root mean square value $\Delta D M_{\text {Host }}=15 \mathrm{pc} \mathrm{cm}^{-3}$. Our analysis shows

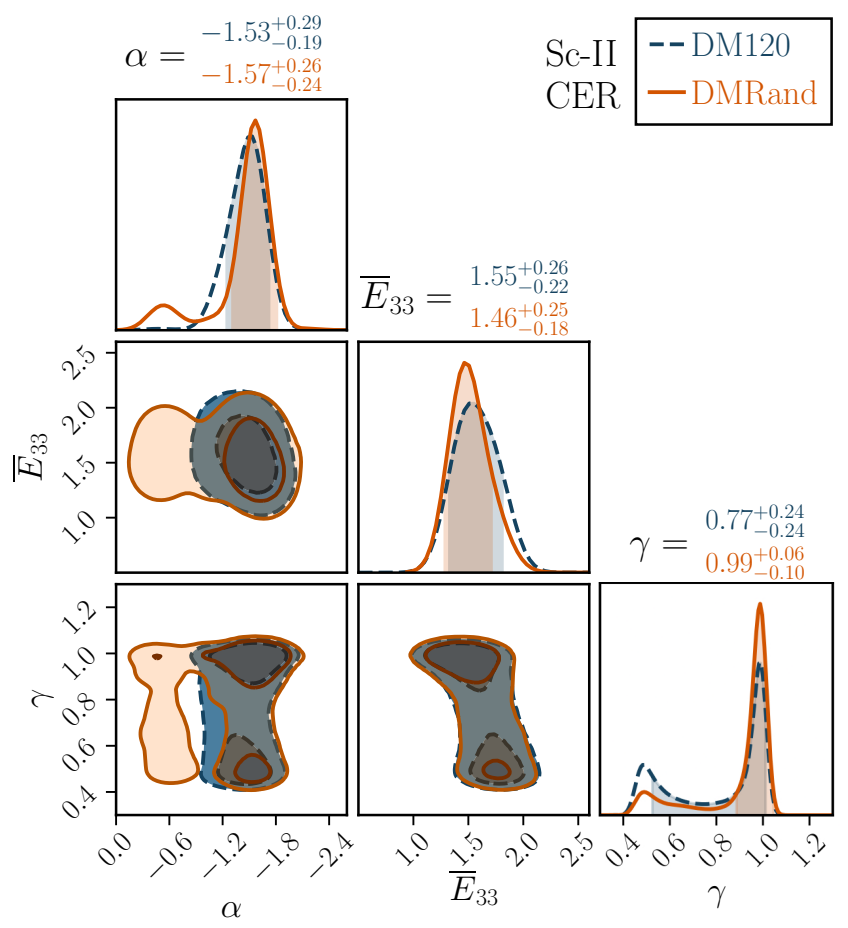

Figure 3. Considering the FRB observations at Parkes, ASKAP, CHIME and UTMOST, this shows the maximum likelihood contours for the parameters $\alpha, \bar{E}_{33}$ and $\gamma$ considering models with Sc-II and CER. The 2D plot show both $68 \%$ and $95 \%$ confidence intervals. Only the $68 \%$ confidence interval is shown in the 1D marginalized plots. The best fit values of $\alpha, \bar{E}_{33}$ and $\gamma$ are shown in the figure with blue color for DM120 and orange color for DMRand respectively.

that the model with a fixed $D M_{\text {Host }}$ is slightly preferred, however we find comparable results from both the models. Considering the preferred combination of the event rate distribution and scattering, we have $\left(\alpha, \bar{E}_{33}, \gamma\right)=\left(-1.53_{-0.19}^{+0.29}, 1.55_{-0.22}^{+0.26}, 0.77 \pm 0.24\right)$ and $\left(-1.57_{-0.24}^{+0.26}, 1.46_{-0.18}^{+0.25}, 0.988_{-0.102}^{+0.058}\right)$ for DM120 and DMRand respectively. The Schechter luminosity function approaches a Diracdelta function as the value of $\gamma$ is increased. The relatively low value of $\gamma$ indicates that there is a considerable spread in the values of the FRB energies. We have assumed $w_{i}=1 \mathrm{~ms}$ for the entire analysis presented here. The analysis was repeated for $w_{i}=0.5 \mathrm{~ms}$ for which the change in the best fit values was found to be less than $10 \%$.

An earlier work (Paper II) had used the KS test to rule out the parameter range $\alpha>4$ and $\bar{E}_{33}>60$ with $95 \%$ confidence. We have checked that the best fit parameter values obtained here are well within the allowed parameter range identified in Paper II.

The results of this paper are expected to provide inputs for any physical model for the nature of the FRB sources and their cosmological distribution. It also throws some light on the effect of scattering in the IGM. We plan to carry out a similar analysis using the recently released catalogue of 492 FRBs detected at CHIME. We expect tighter constraints as more FRBs get included in the analysis.

\section{ACKNOWLEDGEMENT}

We acknowledge the Supercomputing facility 'PARAM-Shakti' at IIT Kharagpur established under the National Supercomputing Mis- 
Table 2. Considering the FRB detected at Parkes, ASKAP, CHIME and UTMOST, this shows the best fit values along with $68 \%$ confidence intervals for $\alpha$, $\bar{E}_{33}$ and $\gamma$ for the different models considered here. The maximum values of $\log$-likelihood $[\log (\mathcal{L})]_{\max }$ are also shown for reference. Results are shown for two different models for $D M_{\text {Host }}$ namely DM120 and DMRand.

\begin{tabular}{cccccccccc}
\hline & \multicolumn{3}{c}{ DM120 } & \multicolumn{5}{c}{ DMRand } \\
\hline & FRB & \multicolumn{3}{c}{ Parameter Values } & Value of & \multicolumn{3}{c}{ Parameter Values } & Value of \\
& Rate & $\alpha$ & $E_{33}$ & $\gamma$ & {$[\log (\mathcal{L})]_{\max }$} & $\alpha$ & $\bar{E}_{33}$ & $\gamma$ & {$[\log (\mathcal{L})]_{\max }$} \\
\hline \multirow{2}{*}{ Sc-I } & CER & $-0.50_{-0.27}^{+0.25}$ & $2.18_{-0.29}^{+0.29}$ & $0.96_{-0.40}^{+0.04}$ & -129.64 & $-0.58_{-0.15}^{+0.19}$ & $2.19_{-0.25}^{+0.27}$ & $0.89_{-0.52}^{+0.12}$ & -133.08 \\
& SFR & $-26.0_{-1.6}^{+1.8}$ & $4.82_{-0.76}^{+0.98}$ & $0.31_{-0.11}^{+0.38}$ & -235.57 & $-25.8_{-1.5}^{+1.7}$ & $5.01_{-0.84}^{+0.86}$ & $0.26_{-0.05}^{+0.40}$ & -235.39 \\
\hline \multirow{2}{*}{ Sc-II } & CER & $-1.53_{-0.19}^{+0.29}$ & $1.55_{-0.22}^{+0.26}$ & $0.77_{-0.24}^{+0.24}$ & -78.39 & $-1.57_{-0.24}^{+0.26}$ & $1.46_{-0.18}^{+0.25}$ & $0.99_{-0.10}^{+0.06}$ & -79.95 \\
& SFR & $-27.5_{-1.7}^{+1.5}$ & $3.96_{-0.70}^{+0.76}$ & $0.37_{-0.16}^{+0.41}$ & -233.31 & $-27.5_{-1.6}^{+1.5}$ & $4.03_{-0.68}^{+0.63}$ & $0.29_{-0.05}^{+0.43}$ & -233.18 \\
\hline \multirow{2}{*}{ No-Sc } & CER & $-2.26_{-0.20}^{+0.19}$ & $1.09_{-0.24}^{+0.19}$ & $0.56_{-0.12}^{+0.34}$ & -94.82 & $-2.25_{-0.15}^{+0.12}$ & $1.09_{-0.08}^{+0.07}$ & $0.47_{-0.02}^{+0.33}$ & -95.29 \\
& SFR & $-30.0_{-0.0}^{+1.1}$ & $2.81_{-0.56}^{+0.61}$ & $0.40_{-0.16}^{+0.49}$ & -229.16 & $-30.0_{-0.0}^{+1.2}$ & $2.80_{-0.56}^{+0.58}$ & $0.40_{-0.17}^{+0.50}$ & -229.34 \\
\hline
\end{tabular}

sion (NSM), Government of India and supported by Centre for Development of Advanced Computing (CDAC), Pune. Some part of the statistical analysis for this work was done using the computing resources available to the Cosmology with Statistical Inference (CSI) research group at IIT Indore.

\section{DATA AVAILABILITY}

The data and codes underlying this article will be shared on reasonable request to the corresponding author.

\section{REFERENCES}

Agarwal D., Lorimer D. R., et al., 2019, MNRAS, 490, 1 Aghanim N., Akrami Y., et al., 2020, A\&A, 641, A12 Amiri M., Bandura K., et al., 2019, Nature, 566, 230 Amiri M., Andersen B. C., et al., 2021, arXiv preprint, 2106.04352 Autcha A., 2014, Science \&; Technology Asia, 19, 14 Bannister K. W., Shannon R. M., et al., 2017, ApJ Letters, 841, L12 Bannister K. W., Deller A. T., et al., 2019, Science, 365, 565 Bera A., Bhattacharyya S., et al., 2016, MNRAS, 457, 2530 Bhandari S., Keane E. F., et al., 2018a, MNRAS, 475, 1427 Bhandari S., Caleb M., et al., 2018b, The Astronomer's Telegram, 12060, 1 Bhandari S., Bannister K. W., et al., 2019, MNRAS, 486, 70 Bhandari S., Sadler E. M., et al., 2020, ApJ Letters, 895, L37 Bhat N. D. R., Cordes J. M., et al., 2004, ApJ, 605, 759 Bhattacharyya S., Bharadwaj S., 2021, MNRAS, 502, 904 Bochenek C. D., Ravi V., et al., 2020, Nature, 587, 59 Burke-Spolaor S., Bannister K. W., 2014, ApJ, 792, 19 Caleb M., Flynn C., et al., 2017, MNRAS, 468, 3746

Caleb M., Spitler L. G., Stappers B. W., 2018, Nature Astronomy, 2, 839 Champion D. J., Petroff E., et al., 2016, MNRAS: Letters, 460, L30 Cordes J. M., Lazio T. J. W., 2003, arXiv preprint, astro-ph/0207156 Farah W., Flynn C., et al., 2018, MNRAS, 478, 1209

Farah W., Flynn C., et al., 2019, MNRAS, 488, 2989

Foreman-Mackey D., Hogg D. W., et al., 2013, PASP, 125, 306-312

Goodman J., Weare J., 2010, Comm. App. Math. Com. Sc., 5, 65

Gupta V., Bailes M., et al., 2020, The Astronomer's Telegram, 13788, 1

Heintz K. E., Prochaska J. X., et al., 2020, ApJ, 903, 152

Houben L. J. M., Spitler L. G., et al., 2019, A \& A, 623, A42
James C. W., Prochaska J. X., et al., 2021, arXiv preprint, 2101.08005 Keane E. F., Kramer M., et al., 2011, MNRAS, 415, 3065

Keane E. F., Johnston S., et al., 2016, Nature, 530, 453

Li C. K., et al., 2020, arXiv preprint, 2005.11071

Lorimer D. R., Bailes M., et al., 2007, Science, 318, 777

Lu W., Piro A. L., 2019, ApJ, 883, 40

Macquart J. P., Koay J. Y., 2013, ApJ, 776, 125

Macquart J. P., Shannon R. M., et al., 2019, ApJ Letters, 872, L19

Macquart J. P., et al., 2020, Nature, 581, 391

Madau P., Dickinson M., 2014, Annual Review of A \& A, 52, 415

Margalit B., Beniamini P., et al., 2020, ApJ Letters, 899, L27

Palaniswamy D., Li Y., Zhang B., 2018, ApJ Letters, 854, L12

Petroff E., Bailes M., et al., 2015, MNRAS, 447, 246

Petroff E., Burke-Spolaor S., et al., 2017, MNRAS, 469, 4465

Petroff E., Oostrum L. C., et al., 2019, MNRAS, 482, 3109

Platts E., Weltman A., et al., 2019, Physics Reports, 821, 1

Price D. C., et al., 2018, The Astronomer's Telegram, 11376, 1

Prochaska J. X., Zheng Y., 2019, MNRAS, 485, 648

Prochaska J. X., et al., 2019, Science, 366, 231

Qiu H., Bannister K. W., et al., 2019, MNRAS, 486, 166

Rafiei-Ravandi M., et al., 2021, arXiv preprint, 2106.04354

Ravi V., Shannon R. M., Jameson A., 2015, ApJ Letters, 799, L5

Ravi V., et al., 2016, Science, 354, 1249

Ridnaia A., et al., 2021, Nature Astronomy, 5, 372

Schechter P., 1976, ApJ, 203, 297

Shannon R. M., et al., 2018, Nature, 562, 386

Shannon R. M., Kumar P., et al., 2019, The Astronomer's Telegram, 12922 Spitler L. G., Cordes J. M., et al., 2014, ApJ, 790, 101

Tavani M., et al., 2021, Nature Astronomy, 5, 401

Thornton D., et al., 2013, Science, 341, 53

Zhang S. B., Hobbs G., et al., 2019, MNRAS: Letters, 484, L147

Zhang S. B., et al., 2020, ApJ Supplement Series, 249, 14

Zhang R. C., Zhang B., et al., 2021, MNRAS, 501, 157 